\title{
Expanding treatment options and ongoing challenges for urologic cancers
}

\author{
Jane de Lartigue, $\mathrm{PhD}$
}

U rologic cancers are those that form in organs of the urinary and male reproductive systems, the most significant among them being cancers of the bladder, kidney, prostate, and testicles. Collectively, they are diagnosed in close to 400,000 Americans each year and are responsible for almost 60,000 deaths annually. ${ }^{1}$ Here, we describe the most recent developments in treating these malignancies.

\section{Prostate cancer: fine-tuning androgen receptor targeting}

Prostate cancer is the most common urologic malignancy, ranking number two in terms of most commonly diagnosed and most lethal cancers in American men. Thanks to effective screening methods, nearly $90 \%$ of prostate cancers are diagnosed while still localized, however, their subsequent clinical behavior is highly variable. In some cases, it is aggressive and metastasizes, whereas in others it is more indolent and can be either readily cured or simply observed. Understanding what drives these distinct outcomes is one of the greatest challenges facing urologic oncologists. ${ }^{2}$

The treatment of prostate cancers that do metastasize has been revolutionized by the understanding that prostate cancer is an androgen-driven disease. Androgens exert their effects through binding to the androgen receptor, which is normally found in the cytoplasm and is maintained in an inactive state through the association of a number of other proteins, including the heat-shock proteins Hsp90, Hsp70, and Hsp40. Androgen binding triggers the androgen receptor's release and it moves into the nucleus and acts as a transcription factor, binding to genes that contain androgen response elements and stimulating their expres- sion. Many of the target genes are involved in the growth, survival, and proliferation of prostate cancer cells (Figure 1). ${ }^{3}$

The cornerstone of treatment for advanced prostate cancer has become surgical castration or medical castration, through the use of androgen deprivation therapy (ADT), which involves treatment with drugs that block the hormone that regulates the syn-

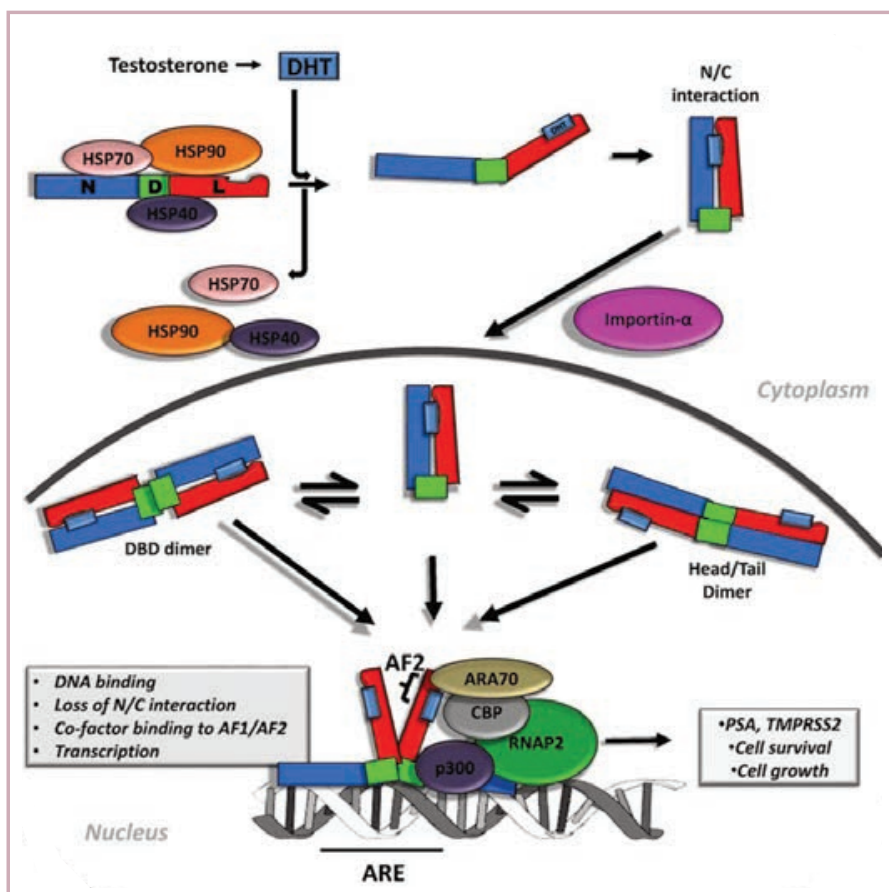

FIGURE 1 The androgen receptor is predominantly found in an inactive state in the cytoplasm in a complex with other proteins, including heatshock proteins (eg, $\mathrm{Hsp} 90, \mathrm{Hsp} 70$, and $\mathrm{Hsp} 40$ ). It is activated upon binding of testosterone or its more active metabolite 5-DHT. Ligand binding releases the androgen receptor from its complex and facilitates N/C dimerization and exposure of a nuclear localization signal that allows the receptor to move into the nucleus. Inside the nucleus it acts as a transcription factor, binding to target genes through androgen response elements. Many of the genes targeted by the androgen receptor are involved in the growth and survival of prostate cancer cells. Reproduced with permission: Lallous N, Dalal K, Cherkasov $A$, et al. Targeting alternative sites on the androgen receptor to treat castration-resistant prostate cancer. Int J Mol Sci. 2013;14(6): 1249612519. 
thesis of testosterone in the testes or that block the body's ability to use androgens. ${ }^{4}$

Although the majority of patients respond initially, the cancer almost invariably becomes castration-resistant prostate cancer (CRPC). Researchers initially believed that CRPC developed the ability to grow independently of androgens, but evidence accumulated that the AR pathway was still very much activated in these cancers. Instead, it seems that tumor cells find alternative ways to active AR signaling, for example, through mutations or amplification of the AR gene or through mutations in the ligand-binding domain that allow the AR to be activated by lower levels of testosterone or by other ligands.., 6

With this knowledge, researchers began to focus their efforts on targeting the AR pathway in more effective ways in CRPC, prompting numerous approvals of drugs with varying mechanisms of action. The more potent and selective AR antagonist enzalutamide was approved in 2012 on the basis of the phase 3 AFFIRM trial in which it demonstrated improved overall survival (OS) compared with placebo. ${ }^{6}$ An alternative way of targeting the AR pathway is through the use of inhibitors of the CYP17A enzyme that plays an important role in androgen synthesis. Abiraterone acetate was approved in 2011 and also significantly improved OS over placebo. ${ }^{7}$ The approval of the first therapeutic vaccine, sipuleucel-T, in 2010 for the treatment of CRPC validated the use of immunotherapy as an effective treatment strategy in prostate cancer. ${ }^{8}$

Despite the expanding treatment options, improvements in OS were modest and patients still ultimately develop resistance, thus there remains a pressing need for new drugs. Ongoing clinical trials are evaluating nextgeneration AR-targeting drugs that are ever more potent and specific and that target other parts of the AR, such as the $\mathrm{N}$-terminal domain rather than the ligand-binding domain. The most clinically advanced of these drugs is the novel anti-androgen ARN-509, for which the phase 3 SPARTAN trial is underway and is expected to complete enrollment sometime this year. ODM-201, a synthetic androgen receptor, is being examined in the phase 3 ATLAS trial, which also began enrolling patients recently. Both of those drugs bind to the AR with higher affinity than enzalutamide.

More specific CYP17A inhibitors are also being tested, and orteronel was looking particularly promising until disappointing phase 3 results prompted discontinuation of its

development program..$^{10}$ Drugs with a combined mechanism of action, both AR antagonist and CYP17A inhibitor, could also prove more effective. The phase 3 ARMOR-SV trial of galeterone, the first in this novel drug class, is underway and expected to complete enrollment in the next 6 months.

\section{Renal cell carcinoma: a hat-trick of recent approvals}

Kidney cancer, most often presenting as renal cell carcinoma (RCC), accounts for about $4 \%$ of all adult malignancies. ${ }^{11} \mathrm{RCC}$ is actually a heterogeneous aggregate of several different subtypes of disease that differ in their histology and clinical course. It can be subdivided into two major groups: clear cell and non-clear cell. Non-clear cell RCC (nccRCC) can be further divided into several other subtypes, including papillary, chromophobe, collecting duct, and renal medullary RCC. ${ }^{12}$

In general, patient outcomes for all types of kidney cancer are poor since it often goes undiagnosed until the advanced stages and is notoriously resistant to chemotherapy. But thanks to genome sequencing studies that 
have vastly improved our understanding of the molecular mechanisms underlying different types of kidney cancer, the situation is steadily beginning to improve, particularly for ccRCC for which there has been a slew of recent drug approvals.

The majority of these tumors exhibit dysregulation of the VHL protein, a component of an E3 ubiquitin ligase complex that plays a central role in "tagging" proteins for destruction by the proteasome. Among its targets is hypoxia-inducible factor 1-alpha (HIF1-alpha), a transcription factor that regulates the cellular response to hypoxia by mediating the expression of a number of different genes, including the vascular endothelial growth factor $(V E G F)$ (Figure 2).

Another frequently altered signaling pathway in ccRCC is the mammalian target of rapamycin (mTOR) pathway, which promotes many of the hallmarks of malignant transformation. mTOR dysregulation is also linked to the VHL pathway, as activation of $\mathrm{mTOR}$ has been shown to increase HIF1-alpha levels and thus stimulate VEGF expression. ${ }^{13,14}$

Drugs designed to target mTOR and VEGF have proved highly effective in the treatment of RCC, realizing substantial gains in progression-free survival (PFS), but only one improves OS and none offers long-term, durable remission. The development of acquired resistance to these targeted therapies is a pressing issue in the design of novel drugs. ${ }^{15}$

Research efforts culminated in three approvals in the past year. Several signaling pathways have been implicated in the development of resistance to VEGF-targeted therapy, among them the hepatocyte growth factor receptor (MET) and fibroblast growth factor receptors (FGFRs) pathways. ${ }^{16}$ Multi-targeted kinase inhibitors have been developed that target both the VEGF receptors and other kinases.

Cabozantinib, for example, includes the MET receptor among its other targets. When compared with the mTOR inhibitor everolimus in the phase 3 METEOR trial, it consistently improved PFS across all subgroups examined, in addition to improving OS. ${ }^{17}$ On the basis of that trial, it was approved by the FDA for the treatment of patients with metastatic RCC who have received previous antiangiogenic therapy.

Targeting the MET receptor may also prove to be a promising therapeutic strategy for a type of nccRCC. Papillary RCC is commonly subdivided into type 1 and type 2 tumors on the basis of histological differences, but genome sequencing studies have suggested the divide is also a molecular one. The majority of type 1 cases are associated with $M E T$ alterations, suggesting they may be susceptible to MET inhibition. Clinical trials testing this hypothesis are ongoing and the results of a phase 2 study of foretinib were recently published, demonstrating an objec- tive response rate of $13.5 \%$ among 74 patients and median PFS of 9.3 months. ${ }^{18,19}$

Meanwhile, lenvatinib targets the platelet-derived growth factor receptor (PDGFR), RET, c-KIT and FGFR 1-4 in addition to the VEGFRs. It was approved in combination with everolimus on the basis of a phase 2 trial in which patients with advanced/metastatic RCC whose disease progressed following VEGF-targeted therapy experienced a significant improvement in PFS when treated with the combination compared with either agent alone. ${ }^{20}$

The third approval was for a very different therapeutic strategy, one that has received significant attention in recent years and revolutionized the treatment of several different cancer types. The immune checkpoint inhibitor nivolumab, which acts to knock out the tumor's ability to hide itself from the immune system, was awarded regulatory approval on the basis of the phase 3 CheckMate-025 trial in which it significantly improved OS compared with everolimus. ${ }^{21}$ These approvals have greatly expanded the second-line treatment options for patients whose disease progresses on VEGF-targeted therapy.

\section{Bladder cancer: immunotherapy yields key approval}

Unlike prostate and kidney cancers, outcomes for patients with bladder cancer have remained stubbornly poor, with 5 -year survival rates of just $15 \%$ among patients with advanced disease. ${ }^{22}$ Since the approval of cisplatin-based combination chemotherapy more than two decades ago, there had been no other FDA-approved treatment options for patients with metastatic disease.

The paucity of effective therapeutic options was not for lack of trying as many different treatment strategies have been tested, including small-molecule inhibitors and monoclonal antibodies targeting the epidermal growth factor receptor (EGFR) and human epidermal growth factor receptor-2 (HER2), both overexpressed in a significant proportion of bladder cancers, fibroblast growth factor receptor (FGFR) inhibitors, since FGFR mutations are commonly seen in this tumor type, and anti-angiogenic drugs, given that the central regulator of angiogenesis, the vascular endothelial growth factor (VEGF) and its receptor are often overexpressed also. Some clinical trials are ongoing (Table 1) but for the most part, have proven disappointing. ${ }^{23,24}$

More recently, the persistence has paid off and a focus on immunotherapy has finally yielded another approved treatment. Cancer cells present a range of unusual proteins on their surface that should serve as antigens, recognizable by the patient's immune system. However, cancer cells are able to suppress this immune response by manipulating the expression of proteins that regulate it, such as the target of atezolizumab, programmed cell death ligand-1 (PD-L1).

The approval for atezolizumab was based on data from 
TABLE 1 A selection of therapies being evaluated in main urologic malignancies

\begin{tabular}{|c|c|c|c|}
\hline Agent & Manufacturer & Mechanism of action & $\begin{array}{l}\text { Stage of clinical testing/ } \\
\text { clinicaltrials.gov identifier }\end{array}$ \\
\hline Seviteronel (VT-464) & $\begin{array}{l}\text { Viamet Pharmaceuticals/ } \\
\text { Innocrin Pharmaceuticals }\end{array}$ & CYP17A inhibitor & Phase 2 (NCT02445976, NCT02130700) \\
\hline $\begin{array}{l}\text { Apalutamide } \\
\text { (JNJ-5602 1927) }\end{array}$ & Janssen Pharmaceuticals & Androgen receptor inhibitor & $\begin{array}{l}\text { Phase } 3 \text { (SPARTAN, NCT02489318; ATLAS, } \\
\text { NCT02531516; NCT02257736) }\end{array}$ \\
\hline EPI-506 & Essa Pharma & $\begin{array}{l}\text { Inhibitor of the androgen } \\
\text { receptor } N \text {-terminal domain }\end{array}$ & Phase 1/2 (NCT02606123) \\
\hline PROSTVAC & Bavarian Nordic & $\begin{array}{l}\text { Vector-based vaccine targeting } \\
\text { prostate-specific antigen }\end{array}$ & Phase 3 (NCT01322490)* \\
\hline DCVAC & Sotio & Dendritic cell-based vaccine & Phase 3 (VIABLE, NCTO2 111577 ) \\
\hline \multicolumn{4}{|l|}{ Renal cell carcinoma } \\
\hline Dovitinib & Novartis & Multitargeted kinase inhibitor & Phase 2 (DILIGENCE-1, NCT01791387) \\
\hline Tivozanib & Aveo Pharmaceuticals & Inhibitors of VEGFR 1-3 & Phase 3 (NCT02627963) \\
\hline Tivantinib & ArQule & MET inhibitor & Phase3 (NCT01688973) \\
\hline Emibetuzumab & Eli Lilly & MET inhibitor & Phase 1/2 (NCT02082210) \\
\hline Crizotinib (Xalkori) & Pfizer & ALK inhibitor & Phase 2 (CREATE, NCTO1524926) \\
\hline Ixazomib (Ninlaro) & Takeda Oncology & Proteasome inhibitor & Phase $1 / 2$ (NCT02447887) \\
\hline Avelumab & Pfizer & Immune checkpoint inhibitor & $\begin{array}{l}\text { Phase } 3 \text { (JAVELIN Renal 101, } \\
\text { NCT02684006) }\end{array}$ \\
\hline Lapatinib (Tykerb) & Novartis & HER2 inhibitor & Phase 2 (NCT02342587) \\
\hline Ramucirumab (Cyramza) & Eli Lilly & VEGFR2 inhibitor & Phase 3 (RANGE, NCT02426125) \\
\hline Cabozantinib (Cabometyx) & Exelixis & Multitargeted kinase inhibitor & Phase 2 (NCTO1688999)* \\
\hline Pembrolizumab (Keytruda) & Merck & Immune checkpoint inhibitor & Phase 2 (KEYNOTE-057, NCT02625961) \\
\hline Nivolumab (Opdivo) & Bristol-Myers Squibb & Immune checkpoint inhibitor & Phase 3 (CheckMate-274, NCT02632409) \\
\hline \multicolumn{4}{|l|}{ Testicular cancer } \\
\hline Veliparib & AbbVie & PARP inhibitor & Phase 2 (NCT02860819) \\
\hline Palbociclib (Ibrance) & Pfizer & Cyclin-dependent kinase inhibitor & Phase 2 (NCT01037790) \\
\hline Pembrolizumab (Keytruda) & Merck & Immune checkpoint inhibitor & Phase 2 (NCT02499952) \\
\hline SGI-110 & Astex Pharmaceuticals & DNMT inhibitor & Phase 1 (NCT02429466) \\
\hline $\mathrm{BBI} 608$ & Boston Biomedical & Cancer stem cell inhibitor & Phase 1/2 (NCT01325441) \\
\hline Brentuximab vedotin (Adcetris) & Seattle Genetics & CD30 antibody & Phase 2 (NCT01851200)* \\
\hline
\end{tabular}


the single-arm phase 2 IMvigor210 trial of patients with locally advanced or metastatic urothelial carcinoma that had progressed during or after platinum-based chemotherapy or within a year of receiving either adjuvant or neoadjuvant platinum-based chemotherapy. A total of 310 patients received a $1,200 \mathrm{mg}$ intravenous dose of atezolizumab on day 1 of 21-day cycles until unacceptable toxicity or progression. The primary endpoint of the study was objective response rate (ORR) and, over a median followup period of 14.4 months, ORR was $26 \%$ among patients with high levels of PD-L1 expression and $18 \%$ for patients with lower expression of this protein. Complete responses were seen in up to $11 \%$ of patients and the median OS was 7.9 months across all patients, and 11.4 months in patients with high PD-L1 expression. Atezolizumab was well tolerated - although $65 \%$ of patients experienced an adverse event (AE), only $11 \%$ were reported to be serious, most commonly involving urinary tract infection, anemia, fatigue, hematuria, and dyspnea. ${ }^{25}$

Further results from a cohort of 119 patients with cisplatin-ineligible disease who were receiving atezolizumab as first-line therapy in the IMvigor210 study were presented at the 2016 annual meeting of the American Society of Clinical Oncology. Among these patients almost a quarter responded to atezolizumab treatment, with median OS of 14.8 months. The fact that this drug was well tolerated in both settings is particularly noteworthy, because bladder cancer tends to occur in older patients who are less able to tolerate the toxicity of chemotherapy. ${ }^{26}$

\section{Testicular cancer: progress limited by heterogeneity}

Testicular cancer is rare, accounting for just $1 \%$ of all malignancies, but its incidence has been increasing in many Western countries in the past several decades and it is the most common solid tumor among men aged 15-35 years. Thanks to its unprecedented sensitivity to chemotherapy in combination with surgical resection, the cure rate for testicular cancer exceeds $90 \%{ }^{27}$

Nonetheless, a proportion of patients develop resistance to chemotherapy and experience relapse or progression despite treatment and the cure rate among these patients

\section{References}

1. UCSF Medical Center. Urologic cancer. UCSF Medical Center Web site. https://www.ucsfhealth.org/conditions/urologic_cancer/. Accessed August 29, 2016.

2. American Cancer Society. Prostate cancer. American Cancer Society Web site. http://www.cancer.org/cancer/prostatecancer/index. Last updated November 3, 2016. Accessed August 29, 2016.

3. Lallous N, Dalal K, Cherkasov A, et al. Targeting alternative sites on the androgen receptor to treat castration-resistant prostate cancer. Int J Mol Sci. 2013;14(6):12496-12519.

4. Brawer MK. Androgen deprivation therapy: a cornerstone in the treatment of advanced prostate cancer. Rev Urol. 2004;6(Suppl 8):S3-S9.

5. Kobayashi T, Inoue T, Kamba T, Ogawa O. Experimental evidence is significantly lower, mostly as a result of limited treatment options.

The molecular mechanisms of resistance to chemotherapy are still poorly understood and the targeted therapies that have been examined as potential treatment options have had limited success. In general, the common mutations in oncogenes and tumor suppressors that are observed in other tumor types are relatively rare in testicular cancers and among the potentially targetable genomic alterations that have been identified, such as mutations in the FGFR, $A K T$ and $P I K 3 C A$ genes, it is unclear what role these might play in the development of chemoresistance, if any. ${ }^{28}$

Some progress is being made in certain types of testicular cancer and several different treatment strategies continue to be evaluated. Among those drugs that look promising in early-stage clinical testing are cyclin-dependent kinases 4 and $6(\mathrm{CDK} 4 / 6)$ inhibitors. These enzymes play a critical role in the regulation of the cell cycle and have emerged as promising drug targets for several different types of cancer. Mature teratomas, tumors that most commonly occur in the testes, have been shown to express high levels of the retinoblastoma protein $(\mathrm{pRb})$. Since $\mathrm{pRb}$ expression is tightly regulated by CDK4/6 it was hypothesized that CDK inhibitors might be effective in the treatment of these tumors.

The results of a phase 2 study of the CDK4/6 inhibitor palbociclib in patients with pRb-expressing germ cell tumors were recently published. Among 29 patients who were evaluable for response, the 24-week PFS rate was $28 \%$ and toxicity was manageable. Benefit was observed mainly in patients with unresectable teratomas and teratomas with malignant transformation. ${ }^{29}$

Cancer stem cells, a group of cells possessing stem celllike properties within the tumor, have been identified in most tumor types and mounting evidence suggests that these cells are hypermalignant and extremely resistant to various current cancer treatments and may be responsible for the metastatic potential of tumors. ${ }^{30}$ As such, they represent an interesting new drug target. The lead candidate BBI-608 targeting the stemness phenotype of these cells is also being evaluated in early-stage clinical trials in testicular cancer. of persistent androgen-receptor-dependency in castration-resistant prostate cancer. Int J Mol Sci. 2013;14(8):15615-15635.

6. Scher HI and Sawyers CL. Biology of progressive, castration-resistant prostate cancer: directed therapies targeting the androgen receptor signaling axis. J Clin Oncol. 2005;23(32):8253-8261.

7. Scher HI, Fizazi K, Saad F, et al. Increased survival with enzalutamide in prostate cancer after chemotherapy. N Engl J Med. 2012;367:1187-1197.

8. De Bono JS, Logothetis CJ, Molina A, et al. Abiraterone and increased survival in metastatic prostate cancer. N Engl J Med. 2011;364:1995-2005.

9. Kantoff PW, Higano CS, Shore ND, et al. Sipuleucel-T immuno- 
therapy for castration-resistant prostate cancer. N Engl J Med. 2010;363(5):411-422.

10. Takeda Pharmaceutical Company Limited. Takeda announces termination of orteronel (tak-700) development for prostate cancer in Japan, USA and Europe. Takeda website. https://www.takeda.com/ news/2014/20140619_6615.html. Published June 19,2014. Accessed August 29, 2016.

11. National Cancer Institute. Genetics of kidney cancer (renal cell cancer). http://www.cancer.gov/types/kidney/hp/kidney-genetics-pdq. Last updated June 30, 2016. Accessed August 29, 2016.

12. Mulgia VF and Prando A. Renal cell carcinoma: histological classification and correlation with imaging findings. Radiol Bras. 2015;48(3):166-174.

13. Su D, Singer EA, Srinivasan R. Molecular pathways in renal cell carcinoma: recent advances in genetics and molecular biology. Curr Opin Oncol. 2015;27(3):217-23.

14. Srinivasan R, Ricketts CJ, Sourbier C, Linehan WM. New strategies in renal cell carcinoma: targeting the genetic and metabolic basis of disease. Clin Cancer Res. 2015;21(1):10-17.

15. Rini BI and Atkins MB. Resistance to targeted therapy in renal cell carcinoma. Lancet Oncol. 2009;10(10):992-1000.

16. Ciccarese C, Di Nunno V, Montironi R, et al. The role of precision medicine in the treatment of metastatic renal cell carcinoma. Exp Rev Precis Med Drug Dev. 2016;1(4):369-377.

17. Chouieri TK, Escudier B, Powles T, et al. Cabozantinib versus everolimus in advanced renal-cell carcinoma. N Engl J Med. 2015;373:1814-1823.

18. The Cancer Genome Atlas Research Network. Comprehensive molecular characterization of papillary renal cell carcinoma. N Engl J Med. 2016;374:135-145.

19. Chouieri TK, Vaishampayan U, Rosenberg JE, et al. Phase II and biomarker study of the dual MET/VEGFR2 inhibitor foretinib in patients with papillary renal cell carcinoma. J Clin Oncol. 2013;31(2):181-186.

20. Motzer RJ, Hutson TE, Glen H. Lenvatinib, everolimus and the combination in patients with metastatic renal cell carcinoma: a randomized, phase 2, open-label, multicentre trial. Lancet Oncol. 2015;16(15):1473-1482.

21. Motzer RJ, Escudier B, McDermott DF, et al. Nivolumab versus Everolimus in Advanced Renal Cell Carcinoma. N Engl J Med. 2015;373:1803-1813.

22. American Cancer Society. Survival rates for bladder cancer. http:// www.cancer.org/cancer/bladdercancer/detailedguide/bladder-cancer-survival-rates. Last updated May 23, 2016. Accessed August 29, 2016.

23. Cheetham PJ and Petrylak DP. New agents for the treatment of advanced bladder cancer. Oncology 2016;30(6):571-579.

24. Carneiro BA, Meeks JJ, Kuzel TM, et al. Emerging therapeutic targets in bladder cancer. Cancer Treat Rev. 2015;41(2):170-178.

25. Rosenberg JE, Hoffman-Censits J, Powles T, et al. Atezolizumab in patients with locally advanced and metastatic urothelial carcinoma who have progressed following treatment with platinumbased chemotherapy: a single-arm, multicentre, phase 2 trial. Lancet 2016;387(10031):1909-1920.

26. Balar AV, Galsky MD, Loriot Y, et al. Atezolizumab (atezo) as firstline (1L) therapy in cisplatin-ineligible locally advanced/metastatic urothelial carcinoma (mUC): Primary analysis of IMvigor210 cohort 1. J Clin Oncol. 34, 2016(suppl; abstr LBA4500).

27. American Cancer Society. Testicular cancer survival rates. American Cancer Society Web site. http://www.cancer.org/cancer/testicularcancer/detailedguide/testicular-cancer-survival-rates. Accessed August 29, 2016

28. Fankhauser CD, Honecker F, Beyer J, Bode PK. Emerging therapeutic targets for male germ cell tumors. Curr Oncol Rep. 2015;17:54-62.

29. Vaughn DJ, Hwang WT, Lal P, et al. Phase 2 trial of the cyclindependent kinase $4 / 6$ inhibitor palbociclib in patients with retinoblastoma protein-expressing germ cell tumors. Cancer 2015;121(9):1463-1468.

30. Kreso A and Dick JE. Evolution of the cancer stem cell model. Cell Stem Cell 2014;14(3):275-291. 